\title{
E20 MPFA for Faults with Crossing Layers and Zig-zag Patterns
}

\author{
G. T. Eigestad ${ }^{a}$, I. Aavatsmark ${ }^{b}$, E. Reiso ${ }^{b}$, H. Reme ${ }^{b}$

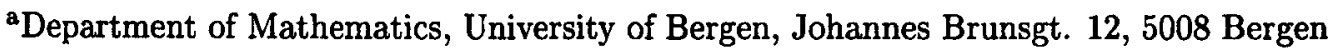 \\ bNorsk Hydro Research Centre, Sandsli, 5020 Bergen
}

\begin{abstract}
In the last years the MPFA control-volume method has been developed for reservoir simulation on general grids. This method gives reliable results for simulation of field cases with heterogeneous, anisotropic media and skew grids. The MPFA method has recently been extended to account for 3D grids where adjacent cells do not share corners. The method has so far been limited to non-crossing layers. In this paper the method is extended to also handle faults with crossing layers, ie. cases where the lateral grid lines of adjacent cells may intersect. This leads to calculations of transmissibility coefficients for general polygonal interfaces. With this extension, virtually all faults in the corner point grid description can be handled by the MPFA method.

Further, the paper discusses faults described by zig-zag patterns and faults aligned with the lateral grid lines.
\end{abstract}

\section{Introduction}

Control volume formulations are often used in the field of porous media flow. Both the ground water branch and the oil industry apply these discretizations for accurate simulations of multiphase flow.

The Multi Point Flux Approximation (MPFA) control volume methods are discretization methods based on continuity of flux and (relaxed) potential continuity. The methods have been derived for structured grids in $2 \mathrm{D}$ and $3 \mathrm{D}$ $[1],[2],[3],[4]$. The latest works published by Aavatsmark et. al. [5], [6] have extended the methods to 2 and 3D faults, where adjacent grid cells do not share corners.
Different types of corners for faulted 3D quadrilateral grids, are classified according to the number of cells that meet in a corner of a grid block [6]. Surrounding each corner, a local interaction is defined, and transmissibilities for sub-interfaces are calculated. From 5 to 8 grid cells meet in a corner, and because of the different interpolations [6], the flux molecules for the sub-interfaces of the interaction region involve from 8 to 11 cells.

This paper will deal with faults where the lateral grid lines of adjacent control volumes are allowed to intersect. A new type of corner then arises. A general 3D corner point simulation grid contains this new type of corners in addition to the corners presented in [6]. Hence, all corners can be handled by the MPFA method, and a wide range of faults can be included in simulations.

The theoretical foundation for the transmissibility calculations are discussed in Sec. 2 . The transmissibility calculations here differ somewhat from previous work. We further discuss physical principles that the discretization must obey. A synthetic numerical example is given in Sec. 3, where the proposed discretization is applied. Sec. 4 discusses zig-zag patterns of $2 \mathrm{D}$ faults, and numerical results are given.

\section{Crossing layers}

The MPFA-method is extended to account for fluxes across sub-interfaces of cell surfaces that arise when lateral grid lines are allowed to intersect. Such a case is depicted in Fig. 1a.

A new corner is defined at the point where lateral grid lines intersect. Such a corner will be termed an irregular corner. Surrounding this corner, an interaction region may be defined, 


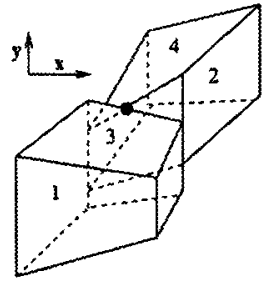

a. Crossing grid lines

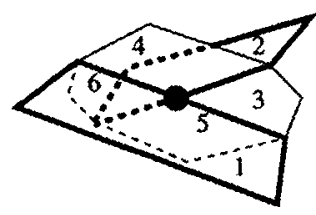

b. Sub-interfaces
Figure 1. Lateral grid lines of cells intersect. (a) Roofs of cells 1 and 2 intersect. Cell 3 is the cell above 1 ; cell 4 is the cell above cell 2 . (b) Irregular corner and sub-interfaces associated with the interaction region. Sub-interfaces are quadrilaterals obtained by a proper sub-division of entire interfaces. Vertical sub-interfaces marked with thin lines, lateral sub-interfaces marked with thick lines.

which contains 4 grid cells. 6 sub-interfaces must be handled for this type of interaction region. The sub-interfaces will be quadrilaterals, and are parts of whole interfaces that separate adjacent cells. The whole interfaces may have 3 to 6 edges. Four of the sub-interfaces of the interaction region will be parts of side surfaces of grid cells, and are numbered 3-6 in Fig. 1b. In the corner point setting these surfaces will lie in the same bilinear surface. The two other sub-interfaces, which are numbered 1 and 2 in Fig. 1b, belong to top or bottom surfaces of the grid cells in question. In this discussion we will allow the top and bottom surfaces of the grid cells to be bilinear, whereas side surfaces of cells will be planar. We hence refer to a vertical plane for four of the sub-interfaces belonging to the irregular corner.

The same idea as for the O-method is applied to derive transmissibilities for the sub-interfaces contained in this interaction region. The potential in each of the four interacting cells is assumed to vary linearly in $\boldsymbol{x}$, (where $\boldsymbol{x}$ is the position vector defined in the cell). This leaves 12 degrees of freedom for determining the transmissibilities for the 6 sub-interfaces.

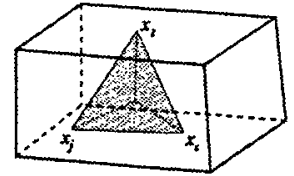

Figure 2. Variational tetrahedron of a grid cell.

\subsection{Transmissibility calculations}

Since the potential is assumed to be linear in each part of the cells that belong to the interaction region, the potential for cell $k$ may be written

$U_{k}=\nabla U_{k} \cdot\left(\boldsymbol{x}-\boldsymbol{x}_{k}\right)+u_{k}$.

In the following, we will define variational tetrahedrons inside each of the four interacting cells. Normal vectors $\nu_{k j}$ of (three) tetrahedron edges will be defined below for each cell $k$. For each cell we use the vectors obtained by going from the cell center $x_{k}$ to the three continuity points $\bar{x}_{k j}$ [2], where $j$ is the cyclic local numbering of continuity points. Ie. it is assumed that the points $\ddot{\boldsymbol{x}}_{k j}$ describe a right handed system seen from the tetrahedron vertex $x_{k}$. Such a tetrahedron is illustrated in Fig. 2. Note that two of the continuity points will be on the same vertical side of the cell in question.

The vectors $\nu_{k j}$ are then defined as

$\nu_{k j}=\left(\bar{x}_{k s}-x_{k}\right) \times\left(\bar{x}_{k t}-x_{k}\right)$,

where $j, s, t$ are cyclic. The gradient of the potential of cell $k$ may then be expressed as [2]

$\nabla U_{k}=\frac{1}{T_{k}} \sum_{j} \nu_{k j}\left(\bar{u}_{k j}-u_{k}\right)$.

The potential $u_{k}$ is the potential at the cell center (node), and $\tilde{u}_{k j}$ are the potentials at the continuity points. $T_{k}$ is equal to 6 times the volume of the tetrahedron used for cell $k$.

One can now formulate the flux continuity conditions for each of the 6 sub-interfaces of the interaction region. Assume that the cells on opposite sides of the sub-interface are $k-$ and $k+$ respectively. The flux through interface $i$, calculated via 
the gradient of the potential of cell $k-$ is given by

$$
f_{i, k-}=-\boldsymbol{n}_{i}^{T} K_{k-} \nabla U_{k-} .
$$

Likewise, the the flux through the same interface calculated via the gradient of the potential for cell $k+$ is given by

$f_{i, k+}=-n_{i}^{T} K_{k+} \nabla U_{k+}$.

The flux continuity condition is that these two expressions are equal. For each of the 6 subinterfaces, flux continuity is posed. The flux continuity equations use the value $\bar{u}_{i}$ (numbering corresponding to numbering of sub-interfaces of Fig. 1) at the continuity point of the interface. Hence, continuity of the potential at the dividing points is satisfied through the flux equations.

One would now assume that the system of equations is closed and defines the transmissibilities. However, the interaction region and location of continuity points hide some redundancy. The system of equations is under-determined by at least one equation when the vertical subinterfaces lie in the same plane. (If two and two sub-interfaces have the same area, the system of equations may be under-determined by two equations)

To show the redundancy, the full system of flux equations will be discussed. The system of equations may be written in terms of potentials at continuity points, $\bar{u}_{i}$, and at grid points, $u_{j}$ :

$A v=B u$.

The vector $v=\left[\bar{u}_{1}, \ldots ., \bar{u}_{6}\right]$ hence consists of the potential values at the dividing points, and $u=$ $\left[u_{1}, \ldots, u_{4}\right]$ consists of the potentials at the cell centers (nodes). As for all MPFA methods, the potentials at the dividing points are only used for determining discrete fluxes, and one should hence use the above equation to eliminate them.

It is convenient to introduce scalar coefficients $\omega_{i j k}$ defined by

$\omega_{i j k}=-n_{i} K_{j} \nu_{j k} / T_{j}$.

Index $i$ refers to the local numbering of subinterfaces (in the particular interaction region), $j$ is the cell number and $k$ is the local continuity point seen from the cell center of cell $j$.

The matrices $A$ and $B$ of Eq. (6) are $6 \times 6$ and $6 \times 4$ respectively. We will categorize irregular corners into two different groups. Seen from the plane on which the irregular corner is located (ie. the plane that 4 sub-interfaces lie in), we will distinguish 'right-left' and 'left-right' generated corners. For corners where the vertical subinterfaces lie in a plane parallel to the $x z$-plane, right-left corners are corners for which the right end point of the lateral grid line of the front cell is located under the right endpoint of the lateral grid line of the back cell. Such a corner is depicted in Fig. 1. The local numbering of grid cells is shown in Fig. 1a, and the local numbering of sub-interfaces is shown in Fig. $1 \mathrm{~b}$.

For the case where the corners are right-left generated, the matrix $\mathbf{A}$ is given explicitly by (8).

To reveal the singularity of matrix $\boldsymbol{A}$, it is sufficient to find multipliers such that a weighted row sum of $\boldsymbol{A}$ is zero. To find these multipliers, we may search for coefficients for each of the 6 rows, such that weighted sums of the elements of the columns are zero. If the coefficients are the same for all rows, we have proven that there is a linear combination of rows of $\boldsymbol{A}$ which is zero (and the matrix is hence singular). It will actually be shown that it is possible to form a linear combination of the last 4 rows of $\boldsymbol{A}$ which is equal to zero.

Assume that we for each column $k$ ( $k=$ $1, \ldots, 6)$ look for weights $c_{i}, i=3, \ldots, 6$ such that

$c_{3} A_{3, k}+c_{4} A_{4, k}+c_{5} A_{5, k}+c_{6} A_{6, k}=0$.

Without loss of generality, we can scale the weights such that $c_{3}=1$. For the first column we then obtain

$$
\begin{aligned}
& -\frac{n_{3}{ }^{T} K_{3} \nu_{33}}{T_{3}}-c_{4} \frac{n_{4}{ }^{T} K_{3} \nu_{33}}{T_{3}} \\
& -c_{5} \frac{n_{5}{ }^{T} K_{1} \nu_{13}}{T_{1}}-c_{6} \frac{n_{6}{ }^{T} K_{1} \nu_{13}}{T_{1}}=0 .
\end{aligned}
$$

Since this should be valid for arbitrary volumes $T_{1}$ and $T_{3}$, we can split this equation into two equations. 


$$
\boldsymbol{A}=\left(\begin{array}{cccccc}
\omega_{133}-\omega_{113} & 0 & \omega_{132} & \omega_{131} & -\omega_{111} & -\omega_{112} \\
0 & \omega_{243}-\omega_{223} & -\omega_{222} & \omega_{241} & -\omega_{221} & \omega_{242} \\
-\omega_{333} & \omega_{323} & \omega_{322}-\omega_{332} & -\omega_{331} & \omega_{321} & 0 \\
-\omega_{433} & \omega_{443} & -\omega_{432} & \omega_{441}-\omega_{431} & 0 & \omega_{442} \\
-\omega_{513} & \omega_{523} & \omega_{522} & 0 & \omega_{521}-\omega_{511} & -\omega_{512} \\
-\omega_{613} & \omega_{643} & 0 & \omega_{641} & -\omega_{611} & \omega_{642}-\omega_{612}
\end{array}\right)
$$

$$
-n_{3}{ }^{T} K_{3} \nu_{33}=c_{4} n_{4}^{T} K_{3} \nu_{33},
$$

$-c_{5} n_{5}^{T} K_{1} \nu_{13}=c_{6} n_{6}^{T} K_{1} \nu_{13}$.

Analogously, the following is obtained for columns 2-6:

Column 2:

$-c_{5} n_{5}{ }^{T} K_{2} \nu_{23}=-n_{3}{ }^{T} K_{2} \nu_{23}$,

$c_{4} n_{4}^{T} K_{4} \nu_{43}=-c_{6} n_{6}{ }^{T} K_{4} \nu_{43}$.

Column 3:

$$
\begin{aligned}
n_{3}{ }^{T} K_{2} \nu_{22} & =-c_{5} n_{5}{ }^{T} K_{2} \nu_{22}, \\
n_{3}{ }^{T} K_{3} \nu_{32} & =-c_{4} n_{4}^{T} K_{3} \nu_{32}
\end{aligned}
$$

Column 4:

$$
\begin{gathered}
-n_{3}{ }^{T} K_{3} \nu_{31}=c_{4} n_{4}{ }^{T} K_{3} \nu_{31}, \\
c_{4} n_{4}{ }^{T} K_{4} \nu_{41}=-c_{6} n_{6}{ }^{T} K_{4} \nu_{41} .
\end{gathered}
$$

Column 5:

$$
\begin{gathered}
-n_{3}{ }^{T} K_{2} \nu_{21}=c_{5} n_{5}{ }^{T} K_{2} \nu_{21} \\
c_{5} n_{5}{ }^{T} K_{1} \nu_{11}=-c_{6} n_{6}{ }^{T} K_{1} \nu_{11}
\end{gathered}
$$

Column 6:

$$
\begin{gathered}
c_{4} n_{4}{ }^{T} K_{4} \nu_{42}=c_{6} n_{6}{ }^{T} K_{4} \nu_{42} \\
c_{5} n_{5}{ }^{T} K_{1} \nu_{12}=-c_{6} n_{6}{ }^{T} K_{1} \nu_{12}
\end{gathered}
$$

If these equations have a solution (for $c_{k}, k=$ $3, \ldots, 6), A$ will be singular. In the corner point setting, corners of grid cells in the $k$-direction are defined to lie on straight lines. As mentioned in the introduction, we consider the case where topand bottom edges are bilinear, whereas the corners of each side edge of a surface are assumed to lie in the same plane. The vertical sub-interfaces belonging to the irregular corner in Fig. 1 will hence have parallel normal vectors. From Eqs. (11)-(22) it is then seen that triplets of them will be internally scaled versions of each other (with the possibility that also $0=0$ expressions occur among them). As an example, one may take (11), (16) and (17) and note that they yield the same information about $c_{4}$.

Furthermore, we can find a vector of coefficients such that a linear combination of the rows of $A$ is zero. This is the left eigenvector of $\boldsymbol{A}$ belonging to the zero eigenvalue, and is given by

$c=\left[0,0, c_{3}, c_{4}, c_{5}, c_{6}\right]^{T}$,

where $c_{3}=1$, and $c_{4}, \ldots, c_{6}$ are found from (11)(22).

This type of interaction region is the first one to be discovered where the matrix $A$ exhibits such a singularity. As seen above, the argument relies on the fact that all 4 normal vectors of the sub-interfaces in the vertical plane are parallel. The singularity of $\boldsymbol{A}$ does not imply that transmissibilities cannot be obtained by the MPFA approach. This will be discussed next.

\subsection{Solution procedure}

From (8) and the matrix $B$ in (6), we find that the columns of $B$ are contained in the column space of $\boldsymbol{A}$. The system of equations (6) then has solutions.

To find the general solutions of a linear system

$A v_{i}=b_{i}$,

the non-trivial solution of the homogeneous equation

$\mathbf{A} \alpha=0$

is first found. In addition, a particular solution of the inhomogeneous problem (24) must be determined. The solution of Eq. (25) may be found by for instance the SVD decomposition [7].

We next discuss how to find particular solutions of the non-homogeneous problem Eq. (24). 


\subsubsection{Particular solutions}

The ordinary interaction regions for the different corners obtained when dealing with 3D faulte have been discussed in [6]. For these cases $\boldsymbol{A}$ nonsingular, and it is possible to express the entials at the dividing points by inverting Eq. ...

$\boldsymbol{v}=\boldsymbol{A}^{-1} \boldsymbol{B} \boldsymbol{u}$

Furthermore, the transmissibility matrix $T$ is then given by

$T=C A^{-1} B-D$.

The matrices $C$ and $D$ can be found similarly to what was done in [6]. Since $A$ is singular for the case with irregular corners, it is not possible to do the inversion in Eq. (26). However, the columns of $\boldsymbol{B}$ are contained in the column space of $\boldsymbol{A}$, and the system (6) then has solutions. We may construct a matrix $V$ by solving Eq. (24) for each of the four columns of $B$.

The matrix $V$ is then $6 \times 4$, with rows $v_{i}, i=$ $1, \ldots, 4$ :

$\boldsymbol{V}=\left[\boldsymbol{v}_{1}\left|\boldsymbol{v}_{2}\right| \boldsymbol{v}_{3} \mid \boldsymbol{v}_{4}\right]$

When $\boldsymbol{A}$ is a rank 5 matrix, the linear dependence of rows of $\boldsymbol{A}$ will occur among the last 4 rows. This means that an LU-decomposition of the matrix is still possible to perform, and this is further used in Eq. (24). If row interchange is allowed in the LU-decomposition, it will always be the case that the last pivot element is zero. Hence, the line corresponding to this element of Eq. (24) simply says $0 \cdot v_{j}=0$. To pick one particular solution, one may replace the zero element of the upper triangular matrix which corresponds to the zero pivot element by 1 (that is; $U(6,6)$ is replaced by 1 if no row interchange occurs).

Because the full solution of Eq. (24) consists of a particular solution and any multiple of the homogeneous solution of Eq. (25), one may form a matrix $E$ to represent the analogue of $A^{-1} B$. The matrix $\boldsymbol{E}$ is given by

$E=\left[v_{1}+d_{1} \alpha\left|v_{2}+d_{2} \alpha\right| v_{3}+d_{3} \alpha \mid v_{4}+d_{4} \alpha\right],(29)$ where the vectors $v_{i}$ are the columns of (28). 4 degrees of freedom are introduced for determining the transmissibility matrix $\boldsymbol{T}$ :

$\boldsymbol{T}=\boldsymbol{C} \cdot \boldsymbol{E}-\boldsymbol{D}$

The matrices $C$ and $D$ are $6 \times 6$ and $6 \times 4$ respectively, and their elements consist of scalar coefficients (7). The rank of $C$ is 4 and the rank of $D$ is 3 .

If the constants are chosen to be $d_{i}=0, i=$ $1, \ldots, 4$, this gives row sums of $T$ that are not zero. In other words, equal potentials of the nodes of the interaction region would not yield zero flux across the sub-interfaces.

It is possible to use the degrees of freedom $\left(d_{i}, 1=1, \ldots, 4\right)$ to make $\boldsymbol{E}$ have the property

$E e_{4}=e_{6}$,

where $e_{4}=[1,1,1,1]^{T}$ and $e_{6}=[1,1,1,1,1,1]^{T}$. When $\boldsymbol{E}$ has this property, straight forward calculations show that the row sums of $\boldsymbol{T}$ are zero, which is one desired property of the transmissibilities.

It turns out that (31) always has solutions, and that only one degree of freedom will be used to insure this. The constraint is then that $d_{1}+d_{2}+$ $d_{3}+d_{4}=D_{t o t}$, where $D_{t o t}$ is the unique fraction given by

$\left(1-\sum_{j} V_{i j}\right) / \boldsymbol{\alpha}_{i}$

This fraction gives the same value for all $i$ when $\alpha_{i}$ is nonzero, and the undefined expression $0 / 0$ when $\alpha_{i}$ is zero. The reason for this (and hence, the reason why Eq. (31) always has solutions) has not been fully understood yet.

\subsection{Multiples of null-space solution}

As there are still three degrees of freedom left in the construction of $\boldsymbol{T}$, we will next discuss possible choices for doing this.

\subsubsection{Physical principles}

The fact that three degrees of freedom are left for determining the transmissibilities through (30), implies that non-unique transmissibilities 
are found for the sub-interfaces of the interaction region around the irregular corner. All these transmissibilities have the property that full flux continuity is satisfied.

It is straight forward to perform tests with different values of the coefficients $d_{i}$ of (29) for different irregular interaction regions with the only constraint that the sum of them is equal to $D_{t o t}$, Eq. (32). These tests show that the transmissibilities can be made arbitrarily small or big when all the four areas of the sub-interfaces in the vertical plane are different. Assuming that the weights $d_{i}$ vary as $D_{\text {tot }} / 4 \pm \beta$, it is seen that the transmissibilities vary linearly with $\beta$. For the subinterfaces 3-6 in the vertical plane, the proportionality factor is columnwise the same for all four interfaces, but different for the 'middle' subinterfaces (no. 1 and 2).

Intuitively this may have unfortunate effects. One consequence is that for fixed potential distributions, the flux calculated across sub-interfaces will be different depending on the choice of weights $d_{i}$. Although flux continuity is assured through the derivation of the transmissibilities, the discrete fluxes may be inaccurate, or even incorrect. (This is related to the choice of fixed potential at one of the continuity points which our modified LU decomposition corresponds to.)

The no-flow constraint for equal potentials at grid nodes is not sufficient to 'fix' our transmissibilities by multiples of the homogeneous solution. The no-flow constraint actually allows the flux to be only $\mathcal{O}(1)$ accurate.

Exact reproduction of uniform flow has been investigated in work by Russell et. al. [9]. When the flow is uniform, the potential will vary linearly in the given direction. This will be the basis for using our degrees of freedom.

To limit the discussion, we will consider the transmissibility calculations for the interaction region corresponding to the irregular corner in Fig. 3. The areas of sub-interfaces 4 and $5, A_{4}$ and $A_{5}$, are equal. This is a representative interaction region for our numerical example in Sec. 3. The nodes of the interacting grid cells are the geometric centres of the cells, and here lie in a plane parallel to the $y z$-plane. Node 1 and 3 have the same $y$-coordinate, and node 2 and 4 have the

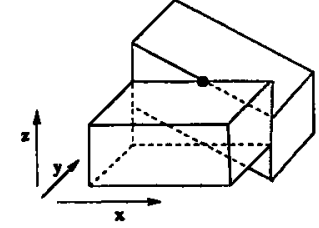

Figure 3. Cells that have intersecting lateral grid lines. Two of the vertical interfaces that touch upon the irregular corner have the same area.

same $y$-coordinate.

For this case, inspection shows that the transmissibilities of the sub-interfaces in the vertical plane do not vary when the weights $d_{i}$ vary. Furthermore, when the pressures at the nodes are given by $\boldsymbol{u}^{\prime}=[1,1-h, 1,1-h]$, where $h$ is a given parameter, the flow is uniform in the direction $(0,1,0)$. The discrete fluxes are exact for these 4 sub-interfaces when applying the transmissibility matrix on $\boldsymbol{u}^{\prime}$.

The middle sub-interfaces (1-2) have normal vectors that are orthogonal to the flow direction, and the analytical fluxes across the sub-interfaces are zero.

Arbitrary weighting may lead to non-zero flux across the interface not parallel to the $x y$-plane; sub-interface 2 here.

For this sub-interface, we will hence use the constraint that the flux is zero when the potential distribution is $u^{\prime}$. It turns out that the weighting should be

$d_{1}=d_{4}=D_{t o t} / 4+\beta, d_{2}=d_{3}=D_{t o t} / 4-\beta$

to make the flux across sub-interface 2 zero. Here it is assumed that the particular solutions $v_{i}(28)$ are orthogonal to the solution $\alpha$ of Eq. (25). Equation (33) also holds for the particular solutions described below Eq. (28), because for these solutions the projections on $\alpha$ are equal for $v_{1}$ and $v_{4}$ and for $v_{2}$ and $v_{3}$.

To determine $\beta$, we must look for other constraints. When $\beta$ now varies, only the transmissibilities corresponding to the middle sub-interface not parallel to the $x y$-plane vary. 


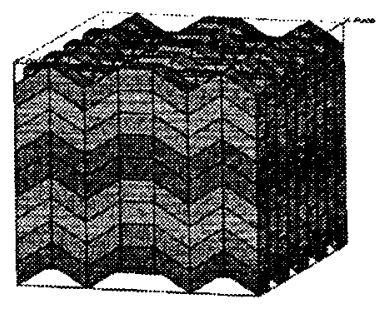

Figure 4. Simulation grid; $7 \times 9 \times 9$.

Although the MPFA approach leads to discretization schemes where the diagonal dominance is weakened compared to the TPFA method, some diagonal dominance must still be demanded (this is physically motivated). In particular, we should demand that the leading transmissibility $\boldsymbol{T}_{22}$ is non-negative.

As the variation of $T$ is linear, we can get an upper or lower bound of $\beta$ in order to make $T_{22}$ of (30) non-negative. The numerical implications when Eq. (33) with bounds on $\beta$ is not satisfied can be quite severe, and we have experienced oscillations in the fronts for two-phase examples.

\section{Numerical results}

A convergence analysis of the suggested discretization has not been performed yet. To validate our results, we will next present numerical results for a two phase flow example (oil and water) in a homogeneous medium. The parameter $\beta$ of (33) is chosen such that no negative leading transmissibilities occur.

Our synthetic grid is $7 \times 9 \times 9$, and is a rather extreme example, where every cell except for the middle $y z$-slize has crossing lateral grid lines (back and front of grid cells), see Fig. 4. A water injector and an oil producer are placed horizontally opposite each other, and it is of interest to study the flow pattern, similarly to what was done in [6]. The reservoir is initially filled with oil, the anisotropy ratio is $k_{v} / k_{h}=1 / 10$, and gravity is discarded.

The simulated saturation pattern after 400 days of fixed injection and production rates is vi-

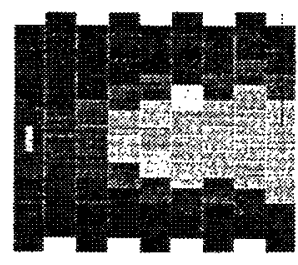

a. MPFA

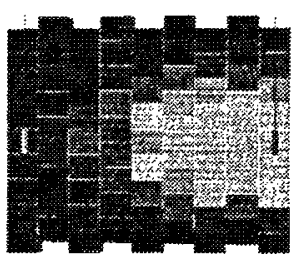

b. TPFA
Figure 5. Water saturation profiles at the same $2 \mathrm{D}$ cross section of the simulation grid after 400 days of production. Homogeneous reservoir, $k_{v} / k_{h}=1 / 10$. (a) MPFA simulation results. (b) TPFA simulation results.

sualized in Fig. 5a.

The same example may be run with a conventional simulator which is based on two-point flux (TPFA) [6], and the saturation profile is visualized in Fig. 5b.

These results fully agree with the results obtained in [6], where the TPFA approach leads to a thicker discus of the water profile. The water arrival at the producer will hence differ significantly for the TPFA-method and the extended MPFA method.

\section{Zig-zag faults versus planar faults}

Simulations on an orthogonal grid with zigiag faults are compared to simulations on a nonorthogonal grid with faults aligned with a grid line. In both cases, the fault plane has the same average orientation laterally. Results computed with MPFA and TPFA are compared. Figure 6 shows the different grid styles, with either one or three faults between an injector and a producer. The orthogonal grids with one or three zig-zag faults will be referred to as "Zigzag-1" and "Zigzag-3", while the non-orthogonal grids with the fault planes aligned with one or three lateral grid lines will be referred to as "Planar-1" and "Planar-3".

The simulation model considered in this study describes a homogeneous, synthetic reservoir, which consists of $10 \times 41 \times 41$ grid blocks, with approximate dimensions $\Delta x=\Delta y=\Delta z=10$ meter. The porosity is 0.3 . The base case is 

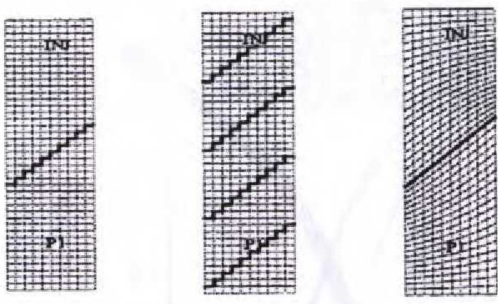

Zigzag-1

Zigzag-3

Planar-1

Planar-3

Figure 6. Simulation grids with zig-zag or planar faults, shown in the lateral plane.

$k_{v} / k_{h}=0.1$, hence there is anisotropy in the vertical direction. Gravity is discarded. For all faults, the gap is $0.5 \Delta z$; this value is chosen according to earlier experience, [5],[6], to maximize the effect of multi-point discretization at faults. Initially the reservoir is filled with oil, and water is injected at one end. The physical well location is unchanged for the individual grids. The simulation results are shown as water cut in the production well. For this simple simulation model, in the comparison of MPFA and TPFA, the effect of zig-zag faults in the grid versus planar faults along a grid line is isolated.

The following parameters are varied in the simulation: Grid style, anisotropy ratio $k_{v} / k_{h}=0.1$ or 0.2 , transmissibility multiplier over the faults is $0.1,0.141,0.2$ or 1.0 . The well location is orthogonal or diagonal in the grid.

We first consider the case of no transmissibility reduction over faults. In the case of only one fault between the wells, see Fig. 7a, there is only a small difference in the results when simulating on the grids Zigzag-1 and Planar-1, using either MPFA or TPFA. For three faults between the wells, grids Zigzag-3 and Planar-3, the effect is more pronounced, see Fig. 7b. In that case it is also clear that for both grid types, MPFA gives the same result. TPFA gives different results, and never the same as MPFA, especially for the zigzag grid.

Fig. 8 shows the effect of increasing $k_{v} / k_{h}$ from 0.1 to 0.2 for the grids with three planar or zigzag faults (Zigzag-3 and Planar-3), for MPFA and

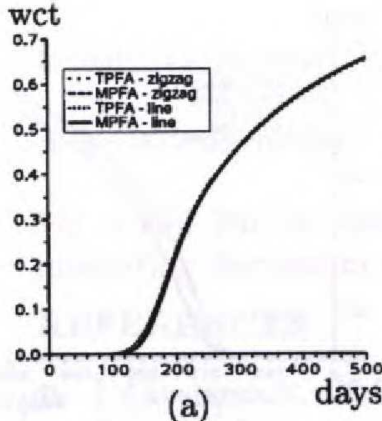

(a)

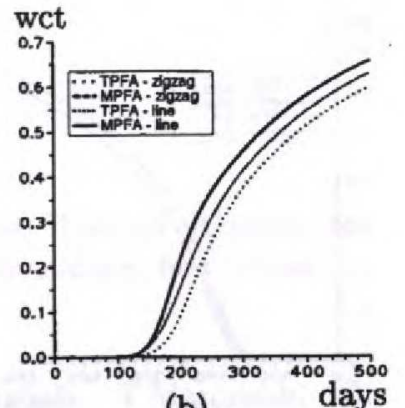

(b)
Figure 7. MPFA vs. TPFA, water cut. (a) Grids Zigzag-1 or Planar-1. (b) Grids Zigzag-3 or Planar-3.

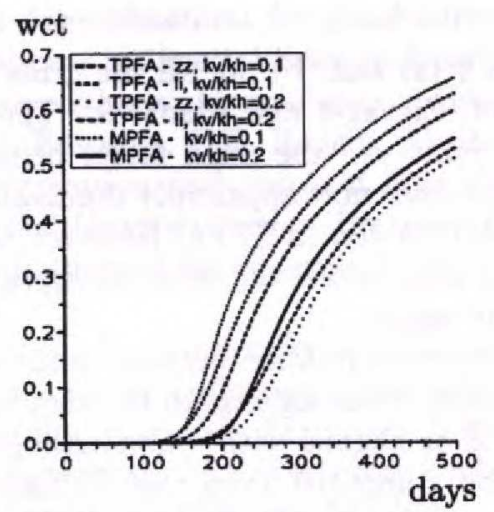

Figure 8. $k_{v} / k_{h}=0.1$ or 0.2 . Grids Zigzag-3 or Planar-3, MPFA vs. TPFA, water cut.

TPFA. The MPFA curves are identical for each value of $k_{v} / k_{h}$, hence only one curve is shown. Increasing the values of $k_{v} / k_{h}$ gives later water breakthrough and lower water cut. The differences in MPFA and TPFA results are smaller for $k_{v} / k_{h}=0.2$ than for $k_{v} / k_{h}=0.1$, for each grid. In the isotropic case with orthogonal grid, MPFA and TPFA give identical results.

Introducing transmissibility reductions over the faults modifies this picture. It is not obvious which transmissibility multiplier value should be used in order to obtain the same flow through a fault described as either zig-zag in an orthogonal grid or aligned with a non-orthogonal grid. Results using the multiplier value 0.1 are shown 


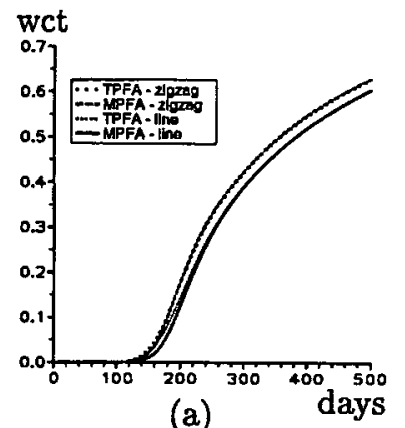

(a)

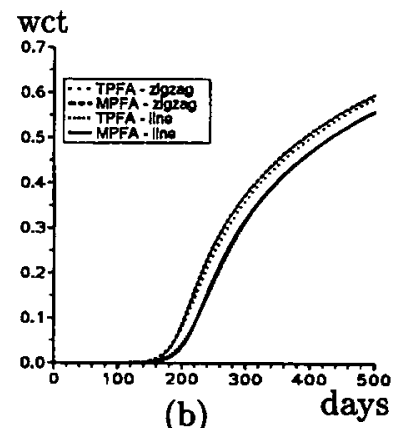

(b)

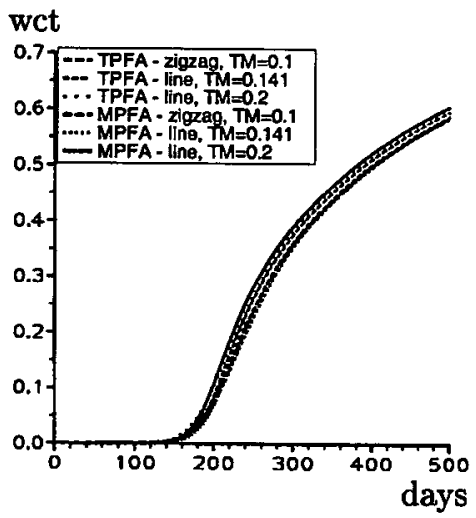

Figure 10. Transmissibility multiplier 0.1 for grid Zigzag-3, multiplier 0.141 and 0.2 for grid Planar3. MPFA vs. TPFA, water cut.

Fig. 10 shows simulation results for MPFA and TPFA, using transmissibility multiplier 0.1 for three zig-zag faults in an orthogonal grid (Zigzag3 ) and transmissibility multiplier 0.141 and 0.2 for three faults aligned with a non-orthogonal grid (Planar-3). For the non-orthogonal grid Planar3, simulations with MPFA where the fault transmissibility multiplier 0.2 was used gave somewhat higher water cut than a corresponding simulation using TPFA. The opposite occurs for a fault transmissibility multiplier of $\sqrt{0.02}=0.141$. For simulations on the orthogonal grid Zigzag-3, with a transmissibility multiplier 0.1 , the water cut curves lie between the other curves, both for TPFA and MPFA. This result indicates that in order to model the fluid flow in a homogeneous, laterally isotropic medium, through a fault that either is described as zig-zag in an orthogonal grid or as aligned with a non-orthogonal grid (the fault plane making an angle of $45^{\circ}$ with the flow direction), the multipliers $m^{*}$ og $m$ in this case should be related by $m^{*}=t \cdot m$, where $t$ has values in the interval $0.141<t<0.2$. However, the result is field dependent, and can not automatically be extended to give general rules for the multipliers $m^{*}$ and $m$. The Pythagoras' relation above may still give an improvement compared to using the same multiplier values for straight and zig-zag faults.

When the wells are located diagonally oppo- 


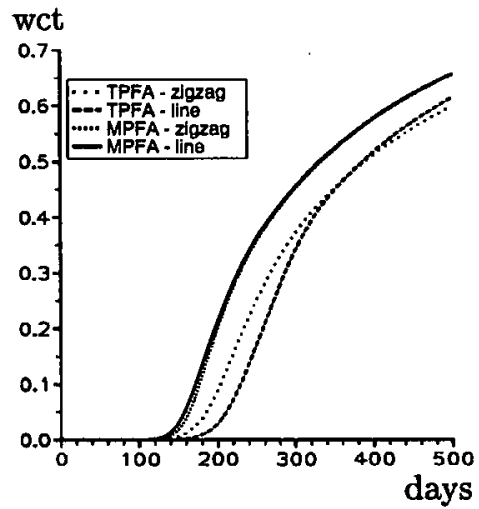

Figure 11. Diagonal well location. No transmissibility reduction, grids Planar-3 or Zigzag-3. MPFA vs. TPFA, water cut.

site each other, rather than parallelly opposite as shown in Figs. 6, increased differences are observed in simulation results, compared to previous results for no transmissibility reduction and $k_{v} / k_{h}=0.1$. Simulated water cut curves are shown in Fig. 11 for grid styles Zigzag-3 and Planar-3, for both MPFA and TPFA. The MPFA curves are almost identical, with a tiny difference at water breakthrough. Also, the difference between MPFA and TPFA curves has increased. However, especially the TPFA water cut curves have changed in character, with larger difference in water breakthrough time and smaller difference at later times. The reason may be that the fluid flow is now modeled as going diagonally in the grid, and the TPFA method suffers from severe grid orientation effects, compared to MPFA. These results are according to [5] and [6].

\section{Conclusions}

The MPFA method has been extended to handle faulted grids with crossing layers. Transmissibilities are calculated for the new sub-interfaces. This makes it possible to simulate fluid flow for many types of faults. Numerical results for synthetic examples are in agreement with previous works. Simulations on grids with zig-zag or planar faults are compared. Fault transmissibility multipliers are evaluated.

\section{Acknowledgments}

We would like to thank Runhild Klausen for interesting discussions on uniform flow issues.

\section{REFERENCES}

1. I.Aavatsmark, T.Barkve, T.Mannseth, $\emptyset$. Bøe: Discretization on non-orthogonal, quadrilateral grids for inhomogeneous, anisotropic media, J. Comput. Phys. 127 (1996), pp. 2-14.

2. I. Aavatsmark: An introduction to Multipoint Flux Approximations for Quadrilateral Grids, To appear in 'Special issue on locally conservative methods', Computational Geosciences.

3. M.G. Edwards, C.F. Rogers: Finite Volume Discretization with Imposed Flux Continuity for the General Tensor Pressure Equation, Computational Geosciences 2, 1998, pp. 259290.

4. S.H. Lee, L.J. Durlovsky, M.F. Lough, W.H. Chen: Finite Difference Simulation of Geologically Complex Reservoirs with Tensor Permeabilities, SPE Reservoir Evaluation \& Engineering 1, 1998, pp. 567-574.

5. I. Aavatsmark, E. Reiso and R. Teigland: Control-volume discretization method for quadrilateral grids with faults and local refinement, Computational Geosciences 5, 2001, pp. 1-23.

6. I. Aavatsmark, E. Reiso, H. Reme and R. Teigland: MPFA for Faults and Local Refinement in $3 D$ Quadrilateral Grids With Application to Field Simulation SPE 66356, SPE Reservoir Simulation Symposium, Houston, Texas, 11-14 February 2001.

7. L.N. Trefethen and D. Bau, III: Numerical Linear Algebra, SIAM Press, Philadelphia, 1997.

8. G. T. Eigestad, R. Klausen. Private communication.

9. R.L. Naff, T.F. Russell, J.D. Wilson: Shape functions for three-dimensional controlvolume mixed finite-element methods on irregular grids, Proceedings, CMWR2002. 